\title{
Zernike Phase Contrast Cryo-EM for Biological Structure Determination
}

\author{
Kazuyoshi Murata*, Xiangan Liu*, Radostin Danev**, Joanita Jakana*, Htet Khant*, Michael F. \\ Schmid*, Kuniaki Nagayama** and Wah Chiu* \\ * National Center for Macromolecular Imaging, Verna and Marrs Mclean Department of Biochemistry \\ and Molecular Biology, Baylor College of Medicine, One Baylor Plaza, Houston, TX 77030 \\ **Okazaki Institute for Integrative Bioscience, National Institute of Natural Sciences, 5-1 Higashiyama, \\ Myodaiji-cho, Okazaki, Aichi 444-8787, Japan.
}

Cryo-EM is capable of revealing structures of large macromolecular assemblies at subnanometer resolution [1]. Structure determination involves extensive data processing of thousands to hundreds of thousands of images due to dose limited noise levels. Zernike phase contrast (ZPC) electron microscopy is a methodology that can enhance the image contrast substantially. The most successful development of this technology is to use a thin carbon film with a central hole of sub-micron diameter as a phase plate [2]. This thin film phase plate is placed in the back-focal plane, with elastically scattered electrons being retarded by the carbon film, but the unscattered central beam passing through the hole unchanged. In the environment of the microscope column, contamination of the phase plates remains one of the greatest challenges. Nagayama and associates developed a method to prevent the phase plate from becoming contaminated in the electron microscope column for a period of time that data can be recorded [2-3]. The phase plate alters the contrast of the microscope, converting normal phase-contrast into amplitude contrast, and increasing the low frequency signals present in the images substantially.

While this technology seemed quite promising at low resolution [3], questions about its capabilities at higher resolution (e.g. $10 \AA$ and beyond) were raised. We have used a 2-D periodic array of bacteriorhodopsin embedded in trehalose as an initial test specimen because a crystalline sample allows us to assess the resolution of the data quite readily without extensive data processing. The images were collected on a JEM2200FS cryomicroscope with a phase plate and a modified objective lens at close-to focus conditions on a Tietz 4k x 4k CCD camera using a low dose system. The quality of each image, containing about 19,000 unit cells, was evaluated by IQ plots of the reflections in Fourier space, after the crystal lattice was unbent to correct for distortion of the crystal. Signal to noise ratios of the diffraction spots are graded from 1 to 8 with 1 being the highest [4]. Figure 1A shows an example of an IQ plot of one crystal image, which illustrates that $90 \%$ of all spots, except for the first order, range in quality from 1 to 4 up to $8 \AA$ resolution. The projection map was calculated by merging the Fourier components in the three images with an imposition of P3 symmetry (Figure 1B). No CTF correction was made for reflections less than $1 / 10 \AA-1$. The resulting map clearly exhibited the projection density of the seven, nearly parallel, membrane-spanning $\alpha$-helices of bacteriorhodopsin in each monomer. This analysis clearly showed that the Zernike phase contrast electron cryomicroscopy can be a subnanometer resolution imaging tool. 


\section{References}

[1]. Glaeser, R.M., Downing, K.H., DeRosier, D., Chiu, W., and Frank, J. Electron crystallography of biological macromolecules. Oxford University Press, Oxford (2007).

[2]. Nagayama, K., and Danev, R. Philos Trans R Soc Lond B Biol Sci 363 (2008) 2153.

[3]. Henderson, R., Baldwin, J.M., Downing, K.H., Lepault, J., and Zemlin, F. Ultramicroscopy 19 (1986) 147.

[4]. Danev, R., and Nagayama, K. J Struct Biol 161 (2008) 211.

[5]. This research has been supported by NIH grant P41RR00250. We thank Dr. J. Spudich at UTHSC for providing the bacteriorhodopsin sample.

A

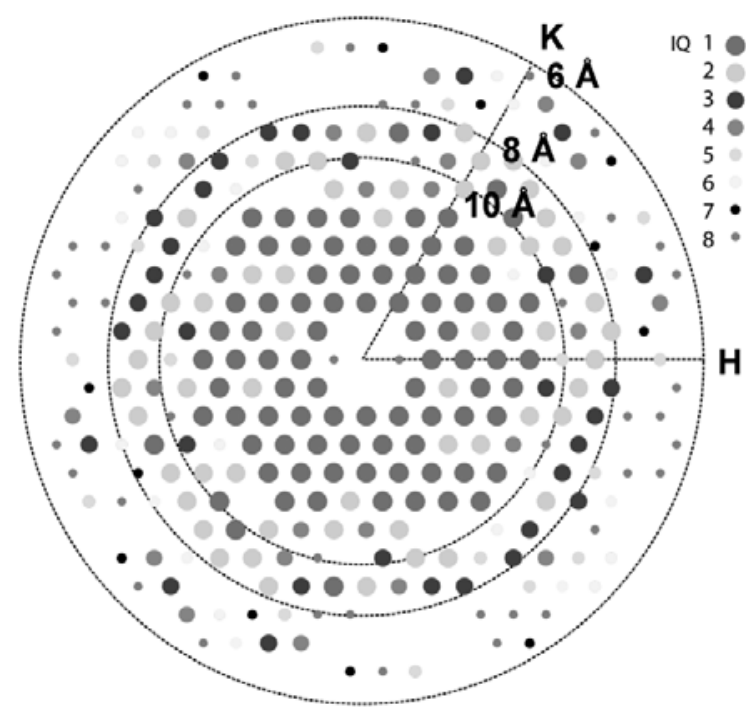

B



Figure 1. Zernike phase contrast imaging of 2-D crystal analysis of bacteriorhodopsin (A) IQ distribution of Fourier diffraction amplitudes calculated from a ZPC image of the 2-D bacteriorhodopsin crystal after computationally correcting the crystal distortion. Different sizes and colors of the dots indicate the peak intensity graded from IQ 1 to 8 . The IQ scale is based on Henderson's convention [3]; $7 \leq$ SNR when IQ 1; $3.5=$ SNR when IQ 2; 1 = SNR when IQ 7; $1<$ SNR when IQ8. Resolution ranges of 10, 8, and $6 \AA$ are indicated with the dashed circles. (B) Projection map of the 2-D bacteriorhodopsin crystal to 8 $\AA$ resolution. The map was calculated from three crystal images by imposing P3 symmetry. Ribbon model of bacteriorhodopsin (PDB ID: 1BRD) is superimposed on the map (Scale bar $20 \AA$ ). 